\title{
An ominous red eye
}

\begin{abstract}
A 49 year-old Malay gentleman presented with a history of a unilateral red eye of one week duration and slowly progressive blurring of vision over a year. On evaluation, he had unilateral anterior chamber inflammation, proptosis, ptosis, ophthalmoplegia and disc swelling. A diagnosis of orbital adnexal lymphoma with systemic nodal involvement and bone marrow infiltration was made following a series of investigations. He was treated systemically with eight cycles of R-CHOP (rituximab, cyclophosphamide, doxorubicin, vincristine and prednisone) therapy and he managed to achieve complete remission. Our aim is to highlight that a unilateral painless red eye simulating conjunctivitis can be an ominous masquerade of orbital lymphoma, especially when there are associated orbital signs (proptosis, external ophthalmoplegia). A high index of suspicion is crucial as prompt diagnosis and timely management can achieve good outcomes.
\end{abstract}

Keywords: orbital lymphoma, uveitis, masquerade, proptosis, ophthalmoplegia
Volume 6 Issue 5 - 2017

\author{
Hah Yan Yee,' Benjamin Chang Chong Ming,,2 \\ Yip Chee Chew, ${ }^{1,2}$ Alvin Wong Seng Cheong, ${ }^{3}$ \\ Thomas Choudary Puttit Srinivasan Sanjay, ${ }^{4}$ \\ 'National Healthcare Group Eye Institute, Singapore \\ ${ }^{2}$ Ophthalmology and Visual Sciences, Singapore \\ ${ }^{3}$ Department of Haematology Oncology, Singapore \\ ${ }^{4}$ Department of Pathology, Singapore
}

Correspondence: Srinivasan Sanjay, Ophthalmology and Visual Sciences, Khoo Teck Puat Hopsital 90 Yishun Central, 768828 Singapore, Tel +65 65558000 ,

Emailsanjay_s@alexandrahealth.ss

Received: March 08, 2017 | Published: April II, 2017
Abbreviations: OAL, orbital adnexal lymphoma; $\mathrm{R}-\mathrm{CHOP}$, rituximab cyclophosphamide doxorubicin vincristine and prednisolone; $\mathrm{LDH}$, lactate dehydrogenase; CT, computed tomography; PET, positron emission tomography; MALT, mucosa associated lymphoid tissue

\section{Introduction}

Red eye is a common ocular presentation and is commonly treated in the primary care setting. Common causes include blepharitis, corneal abrasion, foreign body, subconjunctival hemorrhage, conjunctivitis and dry eye. Sight-threatening conditions causing unilateral red eye such as glaucoma, globe trauma, ocular or orbital infection or inflammation are often painful and accompanied by other discernible features and history. A detailed history taking of signs and symptoms coupled with a careful eye examination are hence crucial in differentiating the causes of the red eye. Our case demonstrates a painless unilateral red eye, which may appear benign as an early manifestation of a systemic and severe disease.

\section{Case presentation}

A 49 year-old Malay man presented to a general practitioner for redness of his left eye which was painless. He was given topical antibiotics but the eye redness persisted. He was subsequently referred to our ophthalmology department for further evaluation. His history revisited revealed that he also had blurry vision in the same eye progressively over a year. There was no tearing, discharge, photophobia or history of similar problem before. He was also systemically well. On examination, his best-corrected visual acuity for the affected left eye was $6 / 9$ and was $6 / 6$ on the right eye. He was also noticed to have left eye proptosis and a left sided partial ptosis (Figure 1a; photograph on presentation), which he had not noticed before. Left eye examination showed conjunctival injection, chemosis and anterior chamber inflammation; cells $3+$ and flare $2+($ Standardization of Uveitis Nomenclature Classification). There were no signs of corneal oedema, fibrin or hypopyon. His optic nerve function was full but his left optic disc was slightly swollen with hyperaemia. There were no choroidal folds or obvious retinal or choroidal masses. His intraocular pressure was noted to be high at $31 \mathrm{mmHg}$ in the left eye; as compared to $16 \mathrm{mmHg}$ on the right. His left eye movements were also limited in all directions of gaze. Ophthalmic exam on the right was noted to be normal. Systemic examination discovered enlarged lymph nodes at the submandibular, sublingual and inguinal areas. His parotid gland was also enlarged.

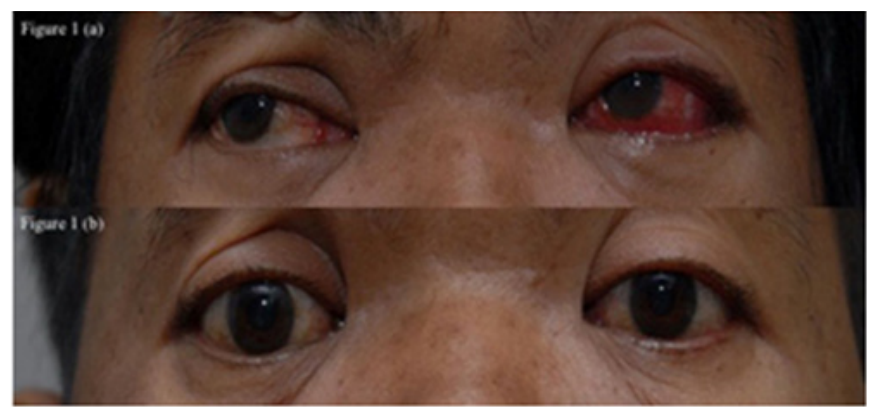

Figure I (a) photograph taken pre-treatment showing left eye conjunctival injection and chemosis (b) photograph taken post-treatment showing complete resolution of conjunctival injection and chemosis in the left eye.

A series of investigations were soon conducted. An ultrasound B-scan (brightness scan) on his left eye showed absence of clinical sign of posterior scleritis (T- sign). Computed tomography scan of orbits and paranasal sinuses showed left proptosis and marked swelling of all the extra-ocular muscles in the left eye. There was no mass lesion seen. Blood tests were not conclusive but revealed high erythrocyte sedimentation rate (ESR) at $80 \mathrm{~mm} / \mathrm{hr}$ and raised white cell count at $11.2 \times 10^{3} / \mathrm{L}$ with lymphocytosis at $51.5 \%$. Thyroid function tests, haemoglobin and platelet levels were normal. The patient subsequently underwent left eye's tenon capsule and superior rectus muscle biopsy for histological diagnosis. The superior rectus muscle histology displayed small B-cell lymphocytic infiltrate with irregular nuclei, indistinct nucleoli and scattered centroblasts (Figure 2; eye muscle histology). Immunohistochemistry showed a predominant CD20+B cell population in the biopsy sample. The tenon's capsule biopsy showed perivascular lymphocytic 


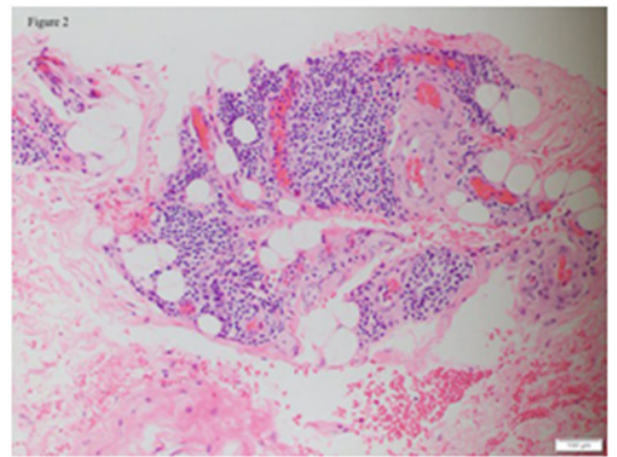

Figure 2 Eye muscle biopsy: Fibro-collagenous tissue with predominant atypical small 'B' cell lymphoid infiltrates and aggregates (Hematoxylin and Eosin, 40X).

Patient was co-managed with the department of haematology and oncology to rule out systemic involvement. Further biopsies were conducted and they took samples from his cervical lymph nodes and bone marrow. His lymph nodes had evidence of marginal zone B-cell lymphoma and suggestion of secondary nodal involvement of an extranodal mucosa-associated lymphoid tissue (MALT) lymphoma (Figure 3 and 4; lymph node histology). His right posterior superior iliac spine bone marrow biopsy was also consistent with B-cell lymphoma infiltrate. There were generalized lymphadenopathy from skull base to inguinal region together with bilateral renal hilar soft tissue infiltration noted on computed tomography of his chest, abdomen and pelvis consistent with the diagnosis of Non-Hodgkin lymphoma. An upper gastrointestinal tract endoscopy was also performed and revealed no gastrointestinal involvement.

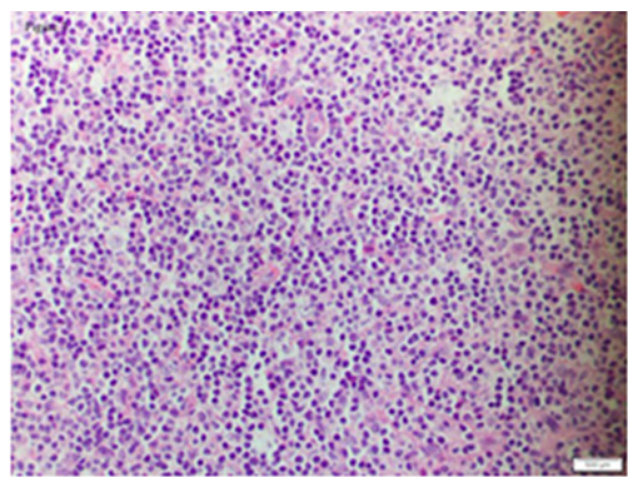

Figure 3 Lymph node biopsy: High magnification image highlighting effaced nodal architecture. The predominant small 'B' lymphoid cells show monocytoid, centrocyte-like and few plasma cells (Hematoxylin and Eosin, I00X).

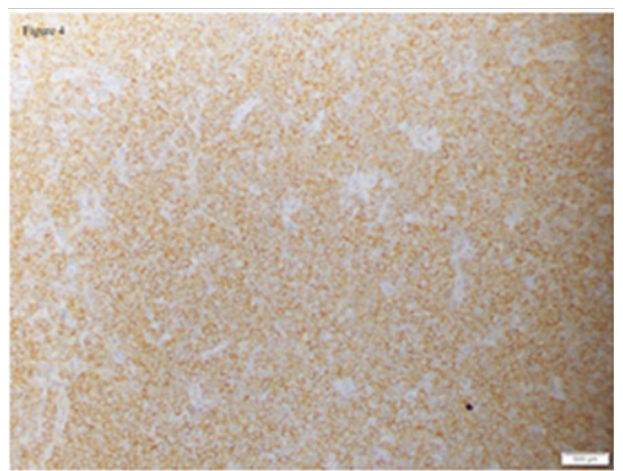

Figure 4 Lymph node biopsy: Immunohistochemical stain of lymph node shows diffuse immunoreactivity for 'B' cell marker (CD20, 40X).
In view of systemic multi-organ involvement of B-cell lymphoma, the patient was promptly started on chemotherapy with immunotherapy by the oncologist. He completed 8 cycles of R-CHOP (rituximab, cyclophosphamide, doxorubicin, vincristine and prednisolone) therapy and managed to achieve complete remission. Upon review six months after his initial presentation, he was asymptomatic with left eye vision of 6/7.5 and a complete resolution of his left proptosis, lid ptosis and disc swelling (Figure 1b; photograph after treatment). In 2017, he has entered his 9 years after his first presentation and he has remained asymptomatic with no sign of relapse.

\section{Discussion}

Orbital adnexal lymphomas (OAL) are the most common primary orbital malignancy in adults 60 years of age and older. ${ }^{1}$ Ocular adnexa comprise of orbit, extraocular muscles, conjunctiva, eyelids, lacrimal gland and apparatus and lymphomas of these structures account for approximately $1 \%$ to $2 \%$ of non-Hodgkin lymphomas and $8 \%$ of extranodal lymphomas. ${ }^{2}$ The majority of cases of OALs are primary extranodal lymphomas; however $10 \%$ to $32 \%$ are secondary tumours in patients with disseminated lymphomas. ${ }^{3-6}$ Ninety-five percent to $100 \%$ of reported cases of orbital adnexal lymphomas are B-cell neoplasms and majority are low grade with extranodal marginal zone lymphoma of mucosa-associated lymphoid tissue (MALT) types being the most common histologic subtype constituting about $35 \%$ to $80 \%$ of cases..$^{7-15}$

Orbital adnexa lymphomas (OAL) are mostly seen in the 5th to 7 th decade of life (in Western and Japanese populations). Our case demonstrates a younger age of presentation for orbital adnexa lymphoma. This is consistent with a few studies in Korean population; which revealed a significantly younger age (median age $\sim 46$ years) at the time of diagnosis. ${ }^{15-17}$ Hence, a further study on our local population with OALs may be useful to better define the age demographics of these patients in Singapore. The risk factors for OAL remain unknown in the majority of cases but studies have shown associations with infections with Chlamydia psittaci. ${ }^{18}$ Chlamydia pneumoniae and Helicobacter pylori. ${ }^{19}$ A cohort study from the United Kingdom showed a significant prevalence of autoimmune thyrotoxicosis with thyroid orbitopathy preceding the diagnosis of OAL. ${ }^{20}$ Genetic abnormalities such as trisomy of chromosome 3 and chromosome 18 have been reported in ocular adnexal MALT lymphoma. ${ }^{21}$ Majority of cases of OALs are primary extranodal lymphomas, however patients with secondary tumours with disseminated tumours may present with initial ophthalmic symptoms. A high index of suspicion for systemic involvement is therefore required and thorough systemic enquiry and clinical examination, chest $\mathrm{x}$-ray, serum lactate dehydrogenase (LDH), b2 microglobulin, chest x-ray, computed tomography (CT) of head and paranasal sinuses, chest, abdomen and pelvis, and bone marrow biopsy are usually performed upon diagnosis of OALs.

Some studies have suggested the use of positron emission tomography (PET) in detection of distant disease as it has higher sensitivity than CT scan in the detection of distant disease (86\% vs $72 \%$ ) in patients with OALs. ${ }^{22}$ The addition of PET imaging to CT and MRI has provided additional, clinically meaningful information that led to "upstaging" and change in patient management in $71 \%$ of cases. ${ }^{23,24}$ However, PET has low sensitivity (27\%) in detecting orbital lesions. ${ }^{15}$ and the use of PET in OALs due to MALT lymphomas are questionable as overall fluorodeoxy glucose (FDG) uptake is reported in only $50 \%$ of cases of MALT lymphomas in other locations. Therefore, it may not be very useful in patients with MALT lymphomas. 
Various treatment modalities are available for the management of patients with OALs including surgical resection, radiotherapy, chemotherapy and immunotherapy with monoclonal antibodies. Radiation therapy is the treatment of choice for the majority of patients with localized OALs whereas combined chemotherapy such as CHOP protocols are reserved for systemic and high grade lymphomas. Rituximab is a monoclonal chimeric anti-CD20 antibody and useful as a single-agent or combined with chemotherapy in treating both newly diagnosed and relapsed disease but early recurrence is common, particularly in pretreated disease.$^{25}$ Our patient in this case is a good example that the treatment with systemic chemotherapy with R-CHOP therapy could potentially achieve complete remission with good prognosis.

\section{Conclusion}

A unilateral red eye could be an early ominous sign for an orbital lymphoma. A high index of suspicion is therefore required in diagnosing OALs especially when there are associated orbital signs (proptosis, external ophthalmoplegia). Early surgical biopsy and appropriate radio-imaging studies are essential for early and adequate diagnosis of orbital and systemic lymphoma. Radiation therapy is the treatment of choice for OALs and combined radio-chemotherapy is reserved for systemic and high-grade lymphomas.

\section{Funding}

None.

\section{Acknowledgments}

Dr Hah Yan Yee and Dr Srinivasan Sanjay had full access to all the data in the study and take responsibility for the integrity of the data and the accuracy of the data analysis.

\section{Conflicts of interest}

Author declares that there is no conflict of interest.

\section{References}

1. Demirci H, Shields CL, Shields JA, et al. Orbital tumors in the older adult population. Ophthalmology. 2016;109(2):243-248.

2. Freeman C, Berg JW, Cutler SJ. Occurrence and prognosis of extranodal lymphomas. Cancer. 1972;29(1):252-260.

3. White WL, Ferry JA, Harris NL, et al. Ocular adnexal lymphoma. A clinicopathologic study with identification of lymphomas of mucosaassociated lymphoid tissue type. Ophthalmology. 1995;102(12):19942006.

4. Fung CY, Tarbell NJ, Lucarelli MJ, et al. Ocular adnexal lymphoma:clinical behavior of distinct World Health Organization classification subtypes. Int $J$ Radiat Oncol Biol Phys. 2003;57(5):1382-1391.

5. Coupland SE, Hellmich M, Auw-Haedrich C, et al. Plasmacellular differentiation in extranodal marginal zone B cell lymphomas of the ocular adnexa:an analysis of the neoplastic plasma cell phenotype and its prognostic significance in 136 cases. Br J Ophthalmol. 2005;89(3):352359 .

6. McKelvie PA, McNab A, Francis IC, et al. Ocular adnexal lymphoproliferative disease:a series of 73 cases. Clin Experiment Ophthalmol. 2001;29(6):387-393.
7. Knowles DM, Jakobiec FA, McNally L, et al. Lymphoid hyperplasia and malignant lymphoma occurring in the ocular adnexa (orbit, conjunctiva, and eyelids):a prospective multiparametric analysis of 108 cases during 1977 to 1987. Hum Pathol. 1990;21(9):959-973.

8. Johnson TE, Tse DT, Byrne GE, et al. Ocular-adnexal lymphoid tumors:a clinicopathologic and molecular genetic study of 77 patients. Ophthal Plast Reconstr Surg. 1999;15(3):171-179.

9. Coupland SE, Krause L, Delecluse HJ, et al. Lymphoproliferative lesions of the ocular adnexa. Analysis of 112 cases. Ophthalmology. 1998;105(8):1430-1441.

10. Jenkins C, Rose GE, Bunce C, et al. Histological features of ocular adnexal lymphoma (REAL classification) and their association with patient morbidity and survival. Br J Ophthalmol. 2000;84(8):907-913.

11. Ferry JA, Fung CY, Zukerberg L, et al. Lymphoma of the ocular adnexa:A study of 353 cases. Am J Surg Pathol. 2007;31(2):170-184.

12. Nakata M, Matsuno Y, Katsumata N, et al. Histology according to the Revised European-American Lymphoma Classification significantly predicts the prognosis of ocular adnexal lymphoma. Leuk Lymphoma. 1999;32(5-6):533-543.

13. Meunier J, Lumbroso-Le Rouic L, et al. Ophthalmologic and intraocular non-Hodgkin's lymphoma:a large single centre study of initial characteristics, natural history, and prognostic factors. Hematol Oncol. 2004;22(4):143-158.

14. Sullivan TJ, Whitehead K, Williamson R, et al. Lymphoproliferative disease of the ocular adnexa: a clinical and pathologic study with statistical analysis of 69 patients. Ophthal Plast Reconstr Surg. 2005;21(3):177-188.

15. Cho EY, Han JJ, Ree HJ, et al. Clinicopathologic analysis of ocular adnexal lymphomas:extranodal marginal zone b-cell lymphoma constitutes the vast majority of ocular lymphomas among Koreans and affects younger patients. Am J Hematol. 2003;73(2):87-96.

16. Woo JM, Tang CK, Rho MS, et al. The clinical characteristics and treatment results of ocular adnexal lymphoma. Korean J Ophthalmol. 2006;20(1):7-12.

17. Yoon JS, Ma KT, Kim SJ, et al. Prognosis for patients in a Korean population with ocular adnexal lymphoproliferative lesions. Ophthal Plast Reconstr Surg. 2007;23(2):94-99.

18. Ferreri AJM, Guidoboni M, Ponzoni M, et al. Evidence for an association between Chlamydia psittaci and ocular adnexal lymphomas. J Natl Cancer Inst. 2004;96(8):586-594.

19. Chan CC, Shen D, Mochizuki M, et al. Detection of Helicobacter Pylori and Chlamydia Pneumoniae genes in primary orbital lymphoma. Trans Am Ophthalmol Soc. 2006;104:62-70.

20. Nutting CM, Shah-Desai S, Rose GE, et al. Thyroid orbitopathy possibly predisposes to late-onset of periocular lymphoma. Eye (Lond). 2006;20(6):645-648.

21. Ferreri AJM, Dolcetti $\mathrm{R}, \mathrm{Du} \mathrm{M}-\mathrm{Q}$, et al. Ocular adnexal MALT lymphoma:an intriguing model for antigen-driven lymphomagenesis and microbial-targeted therapy. Ann Oncol Off J Eur Soc Med Oncol. 2008;19(5):835-846.

22. Sullivan TJ, Valenzuela AA. Imaging features of ocular adnexal lymphoproliferative disease. Eye (Lond). 2006;20(10):1189-1195.

23. Valenzuela AA, Allen C, Grimes D, et al. Positron emission tomography in the detection and staging of ocular adnexal lymphoproliferative disease. Ophthalmology. 2006;113(12):2331-2337. 
24. Gayed I, Eskandari MF, McLaughlin P, et al. Value of positron emission tomography in staging ocular adnexal lymphomas and evaluating their response to therapy. Ophthalmic Surg Lasers Imaging. 2007;38(4):319325 .
25. Ferreri AJM, Ponzoni M, Martinelli G, et al. Rituximab in patients with mucosal-associated lymphoid tissue-type lymphoma of the ocular adnexa. Haematologica. 2005;90(11):1578-1579. 